\title{
Evaluación de las políticas contra el tabaquismo en países latinoamericanos en la era del Convenio Marco para el Control del Tabaco
}

\author{
James Francis Thrasher, PhD, (1,2) Frank Chaloupka, PhD, (1) David Hammond, PhD, (3) \\ Geoffrey Fong, PhD, ${ }^{(4)}$ Ron Borland, PhD, ${ }^{(5)}$ Gerard Hastings, PhD, ${ }^{(6)}$ Michael Cummings, PhD. ${ }^{(7)}$
}

\begin{abstract}
Thrasher JF, Chaloupka F, Hammond D, Fong G, Borland R, Hastings G, Cummings M. Evaluación de las políticas contra el tabaquismo en países latinoamericanos en la era

del Convenio Marco para el Control del Tabaco. Salud Publica Mex 2006;48 supl I:SI55-SI66.
\end{abstract}

\section{Resumen}

Objetivo. El Convenio Marco para el Control del Tabaco (CMCT) pretende coordinar las políticas mundiales que reducen el consumo de tabaco. Con toda probabilidad, estas medidas tendrán efectos en países de ingresos medianos y bajos; no obstante, se requieren estudios de evaluación para determinar sus consecuencias y las posibles sinergias entre las directrices. Material y métodos. El Proyecto Internacional de Evaluación de Políticas para el Control del Tabaco (ITC) es una colaboración internacional para evaluar el efecto psicosocial y conductual que causan las políticas promovidas por el CMCT en adultos fumadores de nueve países. El marco de evaluación ITC utiliza diversos controles de país, con un diseño longitudinal y un modelo conceptual basado en teorías para probar las hipótesis sobre los efectos anticipados de determinadas políticas. Resultados. Los resultados del análisis de datos confirman por lo general los hallazgos anteriores de investigaciones basadas en las recomendaciones del CMCT, y se refieren a las siguientes políticas: promover etiquetas de advertencia con imágenes gráficas, prohibir el uso de las descripciones "ligero" y "suave", establecer ambientes libres de humo de tabaco, incrementar los impuestos, prohibir la publicidad del cigarro e implementar nuevos métodos para evaluar los componen-
Thrasher JF, Chaloupka F, Hammond D, Fong G, Borland R, Hastings G, Cummings $M$.

Evaluating tobacco control policy in Latin American countries during the era of the Framework Convention on Tobacco Control.

Salud Publica Mex 2006;48 suppl I:SI55-SI66.

\begin{abstract}
Objective. The Framework Convention on Tobacco Control (FCTC) aims to coordinate tobacco control policies around the world that reduce tobacco consumption. The FCTC's recommended policies are likely to be effective in low- and middle-income countries. Nevertheless, policy evaluation studies are needed to determine policy impact and potential synergies across policies. Material and Methods. The International Tobacco Control Policy Evaluation Project (ITC) is an international collaboration to assess the psychosocial and behavioral impact of the FCTC's policies among adult smokers in nine countries. The ITC evaluation framework utilizes multiple country controls, a longitudinal design, and a theory-driven conceptual model to test hypotheses about the anticipated effects of given policies. Results. ITC Project results generally confirm previous studies that form the evidence base for FCTC policy recommendations, in particular: the use of graphic warning labels; banning of "light" and "mild" descriptors; smoking bans; increasing tax and price; banning advertising; and using new cigarette product testing methods. Conclusions. Initial findings from the ITC Project suggest that Latin American countries could use similar methods to monitor and evaluate their own tobacco con-
\end{abstract}

(I) Instituto de Investigación en Políticas y Salud, Universidad de Illinois. Chicago, EUA.

(2) Departamento de Investigación sobre Tabaco, Instituto Nacional de Salud Pública. Cuernavaca, Morelos, México.

(3) Departamento de Estudios sobre Salud y Gerontología, Universidad de Waterloo. Canadá.

(4) Departamento de Sicología, Universidad de Waterloo. Canadá.

(5) Consejo para el Cáncer.Victoria, Melbourne, Australia.

(6) Instituto de Mercadeo Social, Universidad de Stirling. Stirling, Escocia, RU.

(7) Instituto Roswell Park de Cáncer. Buffalo, Nueva York, EUA.

Fecha de aprobado: 10 de marzo de 2006

Solicitud de sobretiros: James F.Thrasher. Instituto de Investigación en Políticas y Salud. Universidad de Illinois. 747 West Roosevelt Road, Suite 558. Universidad de Illinois 60608. Chicago, Illinois, Estados Unidos.

Correo electrónico: thrasher@uic.edu 
tes del cigarro. Conclusiones. Los hallazgos iniciales sugieren que los países latinoamericanos podrían aplicar métodos similares para vigilar y evaluar sus propias directrices, así como fortalecer el cuerpo de conocimiento sobre políticas en otros países.

Palabras clave: tabaquismo; política de salud; evaluación de acciones de salud pública; Convenio Marco para el Control del Tabaco; Latinoamérica trol policies while contributing to the evidence base for policy interventions in other countries.

Key words: smoking; health policy; evaluation of public health interventions; Framework Convention on Tobacco Control; Latin America
F 1 Convenio Marco para el Control del Tabaco es un tratado multilateral que promueve una respuesta política internacional coordinada contra la epidemia global del consumo de tabaco. El CMCT se adoptó en 2003, entró en vigor en 2005 y obliga a los países que han ratificado el tratado a observar algunos lineamientos para controlar el tabaco hasta finales de 2009. ${ }^{1}$ El cuadro I resume las principales directrices del CMCT.

Las políticas que promueve el CMCT se basan sobre todo en evidencia de estudios realizados en países de altos ingresos. Es probable que estas mismas medi-

\section{Cuadro I \\ Políticas clave del convenio marco PARA EL CONTROL DEL TABACO}

- Incrementar los impuestos del tabaco

- Proteger a los ciudadanos de la exposición al tabaco en lugares de trabajo, transporte público y lugares públicos cerrados

- Decretar prohibiciones integrales en la publicidad, promoción y patrocinio relacionados con el tabaco

- Regular el empaquetado y etiquetado de los productos del tabaco para evitar el uso de términos confusos y equívocos como "ligeros" y "suaves"

- Regular el empaquetado y etiquetado de los productos del tabaco para asegurar que se comunican advertencias adecuadas sobre el producto a los consumidores, por ejemplo, la obligación de colocar y rotar con frecuencia las advertencias sanitarias y que éstas ocupen al menos un espacio de $30 \%$ de las áreas principales de exhibición en los paquetes de tabaco (de manera ideal $50 \%$ o más) y puedan incluir fotos o pictogramas

- Regular las pruebas y la descripción del contenido y las emisiones de los productos del tabaco

- Promover la conciencia general de aspectos relacionados con el tabaco para asegurar un amplio acceso a programas educativos eficaces e integrales y despertar el interés público en los programas relacionados con el riesgo para la salud que representan el tabaco y la exposición al humo

- Promover e instituir programas eficaces que tengan como propósito cesar el consumo del tabaco

- Combatir el contrabando, incluida la colocación de marcas en los destinos finales de los empaques

- Aplicar legislaciones y programas para prohibir la venta de productos del tabaco a menores de edad

- Instituir políticas para apoyar económicamente las fuentes de ingreso alternativas para los trabajadores del tabaco, los agricultores y los vendedores individuales das reduzcan el consumo de tabaco en países de ingresos medianos y bajos, ya que provocan un incremento de los impuestos sobre los cigarrillos. ${ }^{2}$ Sin embargo, es necesario realizar estudios en este grupo de países para determinar si otras directrices de control del tabaco promovidas por el CMCT tendrán en ellos un resultado similar. Además, aun cuando se sabe que estas políticas reducen el consumo de tabaco en países de alto ingreso, los mecanismos mediante los cuales éstas ejercen un efecto son casi siempre imprecisos. Determinar las vías específicas a través de las cuales los lineamientos disminuyen el consumo de tabaco puede arrojar luz sobre las posibles sinergias entre las diferentes acciones y vislumbrar de qué manera las prácticas de la industria tabacalera pueden comprometer las consecuencias de las políticas contra el tabaquismo.

La institución de las medidas del CMCT alrededor del mundo confiere a los investigadores la extraordinaria oportunidad de coordinar sus esfuerzos para contestar tales preguntas. En este documento se describen el diseño del estudio, el marco conceptual y los resultados preliminares del Proyecto Internacional de Evaluación de Políticas para el Control del Tabaco (proyecto ITC), un esfuerzo internacional de colaboración para evaluar el efecto psicosocial y conductual de las directrices del CMCT entre los adultos fumadores. Hasta el momento actual, ningún país de Latinoamérica se ha adherido al estudio ITC. La evidencia sobre los lineamientos eficaces de control del tabaco se fortalecería con la inclusión de información comparativa de estos países, cuyas distintas historias sociales, culturales y político-económicas pueden incrementar, o tal vez disminuir, el efecto de las políticas antitabaco.

\section{Material y métodos}

\section{El Proyecto Internacional de Evaluación de Políticas para el Control del Tabaco}

El marco de evaluación ITC utiliza múltiples controles de país, un diseño longitudinal y un modelo conceptual basado en teorías para probar hipótesis relacio- 
nadas con efectos anticipados de algunas políticas. En la actualidad el proyecto incluye a cohortes, grupos de fumadores representativos, en nueve países (Australia, Canadá, China, Irlanda, Malasia, Corea del Sur, Tailandia, Reino Unido y Estados Unidos de América) y se obtuvieron muestras representativas de 1000 a 2000 fumadores adultos en cada país, con la previsión de incluir a otros países en el futuro. El modelo conceptual del proyecto se sustenta en teorías de economía, psicología social y comunicación en salud y asume que los mecanismos causales comunes subyacentes pueden explicar la influencia que ejercen en la conducta las distintas políticas relacionadas con el control del tabaco.*

La figura 1 ilustra el marco conceptual general que guía al proyecto ITC. Este modelo de cadena causal sugiere que las variables psicosociales específicas de los lineamientos median entre la influencia de las políticas y las variables resultantes que registran un efecto en la salud pública, como dejar de fumar. La trayectoria entre la exposición a la política y el cambio de la conducta puede ser directa, pero es más común que se presente a través de cambios relacionados con la actitud hacia el hábito de fumar y en las convicciones, variables ambas que predicen de manera consistente las conductas relacionadas con el cigarrillo. Por ejemplo, es probable que

* Fong GT, Cummings KM, Borland R, et al. The conceptual framework of the International Tobacco Control Policy Evaluation Project. Tobacco Control. Documento no publicado. los fumadores tomen consciencia de las advertencias en cajetillas de cigarros antes de pensar en el contenido de éstas. En el plano hipotético se esperaría que los fumadores cambien sus actitudes y expectativas relacionadas con el hábito de fumar. Con base en este marco, los cambios podrían ejercer una influencia en las intenciones de fumar y también en la conducta relacionada con el cigarrillo. En el caso de algunas directrices, pueden existir vías a través de varios tipos de mediadores, ya sea específicos de las políticas o inespecíficos.

Los componentes para evaluar este modelo conceptual permiten probar de modo empírico las vías a través de las cuales surge una hipótesis de trabajo e incluso probar modelos alternativos para el efecto de estas directrices. A este respecto, diversos factores pueden moderar las cadenas causales propuestas. En atención a este marco, las vías de influencia general pueden ser más débiles, por ejemplo, en los fumadores con mayor adicción que en los fumadores con menor. Además, el proyecto ITC se diseñó de manera específica para descartar las explicaciones alternativas sobre las consecuencias de las políticas y por lo tanto se fortalecen las conclusiones sobre los efectos específicos de dichos lineamientos. Los datos del ITC se pueden emplear para probar de forma explícita similitudes y diferencias entre las vías de influencia, directrices y poblaciones. La comprensión detallada de la influencia de una política sobre una conducta determinada puede ayudar a dilucidar las vías de mayor influencia y fortalecer los resultados de salud pública. Por otro lado, conocer estas vías puede llevar a idear nuevas

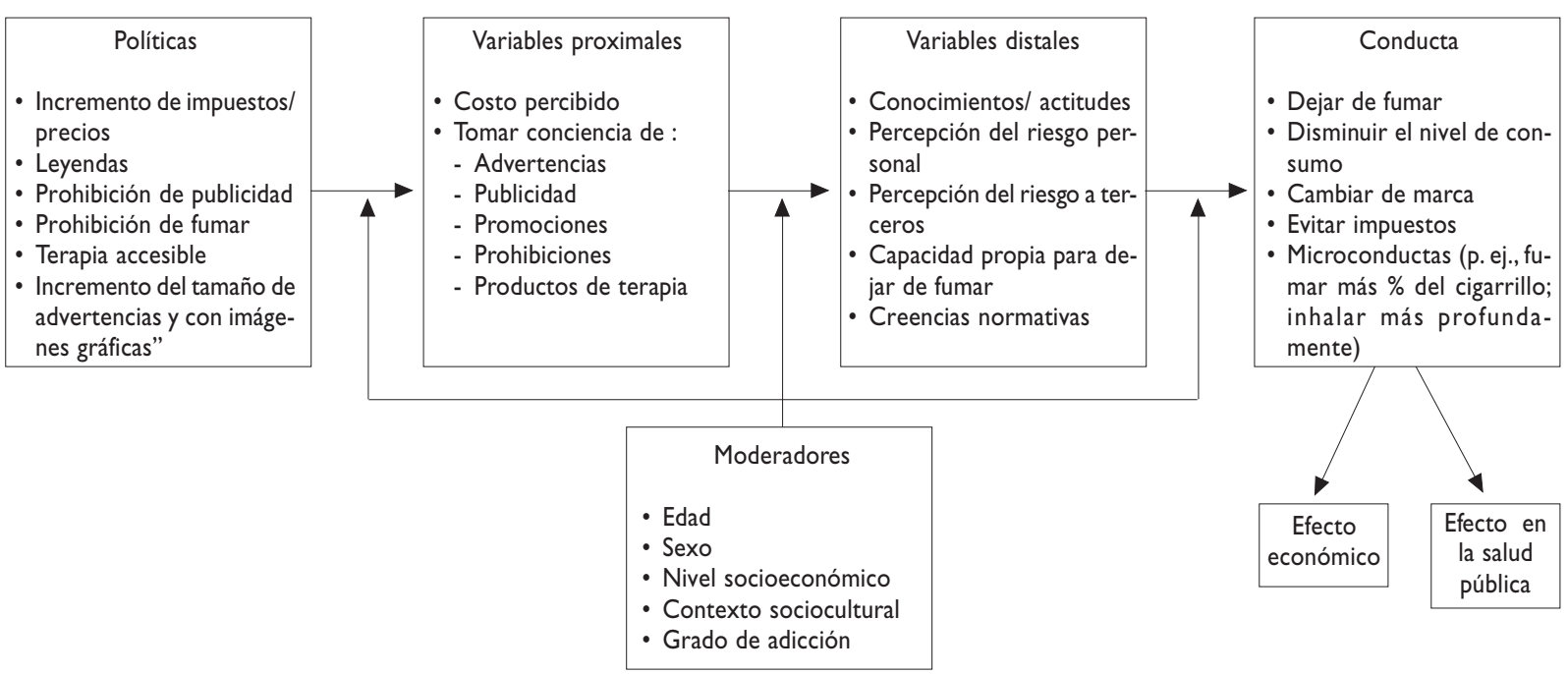

Figura I. Marco conceptual para el Proyecto Internacional de Evaluación de Políticas para el Control del TABAco 
políticas e intervenciones que mejoren el control del tabaco y tengan un efecto sinérgico con las disposiciones que promueve el CMCT.

\section{Diseño y expansión del estudio ITC}

No es posible llevar a cabo un estudio experimental aleatorio para evaluar los efectos de las políticas de control del tabaco, ya que los gobiernos, y no los investigadores, controlan la institución de las directrices. Además, las opciones políticas están relacionadas con frecuencia entre sí, por lo que es imposible casi siempre considerar los lineamientos como sucesos aislados. . La mayoría de los estudios nacionales relacionados con las políticas para el control del tabaco ha utilizado diseños de series de tiempo, repeticiones transversales y series de tiempo pooled con repeticiones transversales que examinan el resultado del interés antes y después de la aplicación de una política. A pesar de que son valiosos, estos estudios tienen poca validez interna debido a la ausencia de un buen grupo de control, por lo que es difícil descartar una influencia de las tendencias seculares y otras directrices e intervenciones que pueden inducir cambios en los patrones del tabaquismo. Para la resolución de estos problemas, el proyecto ITC emplea un diseño semiexperimental con encuestas estandarizadas que permiten hacer comparaciones entre los países que han instituido las medidas y los que no lo han hecho. ${ }^{\ddagger}$ De igual modo, si se establecen cohortes de fumadores adultos en cada nación, los fumadores pueden convertirse en sus propios controles con el paso del tiempo. Modelar las influencias en los cambios individuales en una escala temporal posibilita un mayor control estadístico sobre las posibles variables de confusión que se admitirían al utilizar uno de los diseños estándar. También es posible modelar la cadena causal. Por último, el proyecto ITC permite examinar las diferencias entre los países, incluidas aquellas que pueden atribuirse a los niveles estatal o regional.

El proyecto ITC comenzó en 2002 con el establecimiento de cohortes de representación nacional de aproximadamente 2000 fumadores adultos en Australia, Canadá, Reino Unido y Estados Unidos de América. En esta encuesta, la cohorte original del ITC se ha

\footnotetext{
* Fong GT, Cummings KM, Borland R, et al. The conceptual framework of the International Tobacco Control Policy Evaluation Project. Tobacco Control. Documento no publicado.

‡ Thompson ME, Fong GT, Hammond D, Boudreau C, Dreizen PR, et al. The methodology of the International Tobacco Control Policy Evaluation Project. Tobacco Control. Documento no publicado.
}

\section{Cuadro II \\ Países que participan actualmente en el proyecto INTERNACIONAL DE EVALUACIÓN DE POLÍTICAS PARA EL CONTROL DEL TABACO}

País (año de inicio) Descripción del diseño de la encuesta

Australia (2002) 2000 fumadores adultos encuestados mediante Canadá (2002) entrevista realizada por marcado digital aleatorio Reino Unido (2002) en 2002 y repetida cada año hasta 2008

Estados Unidos

de América (2002)

\begin{tabular}{ll}
\hline Irlanda (2003) & $\begin{array}{l}\text { I } 000 \text { fumadores adultos encuestados mediante } \\
\text { entrevista realizada por marcado digital aleatorio } \\
\text { en } 2003,2004 \text { y } 2005, \text { como respuesta a la ley } \\
\text { sobre ambientes libres de tabaco en Irlanda }\end{array}$ \\
\hline Malasia (2005) & $\begin{array}{l}2000 \text { fumadores adultos y I } 000 \text { adolescentes } \\
\text { no fumadores (entre I3 y I7 años de edad) en- } \\
\text { trevistados en persona, en sus hogares, en 2005, } \\
2007 \text { y } 2009\end{array}$ \\
\hline Tailandia (2005) & $\begin{array}{l}2000 \text { fumadores adultos y I 000 adolescentes } \\
\text { no fumadores (entre I3 y I } 7 \text { años de edad) en- } \\
\text { trevistados en persona, en sus hogares, en 2005, } \\
2007 \text { y } 2009\end{array}$ \\
\hline Corea del Sur (2005) & $\begin{array}{l}\text { I } 000 \text { fumadores adultos encuestados mediante } \\
\text { entrevista realizada por marcado digital aleatorio } \\
\text { en } 2005, \text { con planes de repetir las encuestas cuan- } \\
\text { do se disponga de recursos económicos }\end{array}$ \\
\hline China (2006) & $\begin{array}{l}800 \text { fumadores adultos y } 200 \text { no fumadores de siete } \\
\text { ciudades entrevistados en persona, en sus hoga- } \\
\text { res, en } 2006, \text { con planes de repetir las encuestas } \\
\text { cuando se disponga de recursos económicos }\end{array}$ \\
\hline
\end{tabular}

expandido desde entonces para incluir a fumadores de Irlanda, Malasia, Tailandia, Corea del Sur y China (cuadro II). El proyecto se centra en los fumadores porque ellos son el objetivo principal de la mayoría de las políticas de control del tabaco.

Para reclutar a los fumadores se utilizó la entrevista telefónica con selección aleatoria en la mayoría de los países participantes en el ITC. El método de encuestas personales (cara a cara) se ha usado con éxito en Malasia, Tailandia y China, en donde la distribución desigual de los teléfonos suscitó preocupación acerca del método de selección y el sesgo que acompañaba a la selección telefónica aleatoria en esas naciones. También se han encuestado cohortes de adolescentes en Tailandia y Malasia para comprender los efectos del control del tabaco en la iniciación y evolución del hábito de fumar. Otro de los avances principales del proyecto es el análisis de los componentes del cigarrillo y su diseño entre países, con el fin de determinar si las características del producto influyen en los patrones del consumo de tabaco. 


\section{Resultados}

\section{Hallazgos del proyecto ITC}

Los resultados del análisis de datos del proyecto ITC confirman por lo general los hallazgos anteriores de investigaciones basadas en las recomendaciones del $\mathrm{CMCT}$, en tanto que apoyan los mecanismos a partir de los cuales se formulan hipótesis sobre las consecuencias de estas disposiciones en la conducta de los fumadores. Por ejemplo la exigencia de incluir etiquetas de advertencia con imágenes gráficas en las cajetillas de cigarrillos y la prohibición de utilizar métodos de prueba ISO para determinar los niveles de los componentes de los cigarrillos. Los análisis actuales y continuos revisan los efectos y los mecanismos de influencia en relación con todos los dominios de las directrices del CMCT.

La mayor parte de los hallazgos ITC, hasta la fecha, procede de países de ingresos altos y angloparlantes. El análisis de datos de las cohortes establecidas en fecha reciente en Asia ayudará a determinar si los mecanismos de las políticas y sus efectos difieren en estos países de ingresos medianos y bajos. A continuación se resumen los hallazgos iniciales del estudio ITC relacionados con las etiquetas de advertencia, la creación de espacios libres de fumadores y los impuestos, en un esfuerzo por ilustrar de qué forma el estudio puede registrar el desarrollo de políticas en América Latina y otras naciones (ver cuadro III).

\section{Etiquetas de advertencia en cajetillas de cigarros}

El artículo 11 de la CMCT declara que las etiquetas con advertencias sanitarias en las cajetillas de cigarros: "deberían ocupar $50 \%$ o más de las superficies principales expuestas y en ningún caso menos de 30\% de las superficies principales expuestas". De acuerdo con sugerencias relacionadas con teorías de comunicación para promover la salud y con investigadores del tabaco, ${ }^{6-9}$ las etiquetas de advertencia son un medio de transmisión ideal de mensajes para prevenir el consumo del tabaco porque llegan al público objetivo (es decir, los fumadores y las personas interesadas en fumar) en el momento correcto (al comprar cigarrillos y fumar) y con una elevada frecuencia (20 veces al día para fumadores que consumen una cajetilla diaria). Esta medida puede ser en extremo efectiva en relación con el costo en comparación con otros esfuerzos de comunicación, sobre todo cuando las disposiciones estipulan que la industria tabacalera asuma el costo de impresión de las etiquetas en las cajetillas. Como consecuencia de estas ventajas, las etiquetas de advertencia pueden ayudar a los países a cumplir con el principio básico del CMCT, según el cual "todos deben estar informados de las consecuencias sanitarias, la naturaleza adictiva y la amenaza mortal del consumo del tabaco y de la exposición al humo de tabaco".

La mayoría de los países latinoamericanos todavía no ha alcanzado los estándares de las etiquetas de advertencias; por ejemplo, en un acuerdo voluntario suscrito en mayo de 2004 entre la Secretaría de Salud de México y la industria del tabaco se especificaba que las etiquetas de advertencia deberían incrementar su tamaño y ocupar $50 \%$ de la parte trasera de las cajetillas de cigarrillos, e incluir en el lateral de cada paquete el mensaje: "actualmente no existe un cigarro que reduzca los riesgos a la salud." Como el texto no está en negritas, es relativamente pequeño (12 puntos, con letra helvética normal) y no existe la obligación de incluir una advertencia en la parte frontal del paquete, este acuerdo representa una oportunidad perdida del gobierno mexicano para satisfacer o rebasar los estándares de etiquetas del CMCT. En realidad, los datos longitudinales del ITC confirman los resultados de otras investigaciones, ${ }^{10-11}$ en tanto que indican que las etiquetas con textos en negritas cumplen con los estándares del CMCT y son eficaces. ${ }^{12}$ No obstante, el cambio que no se percibe en las etiquetas de advertencia en México puede ocasionar una disminución del consumo del tabaco. Sin embargo, sin un mecanismo de evaluación en el lugar, además de la valoración del consumo per cápita, no es posible conocer alguna consecuencia de esta política a partir de los efectos de otra, las políticas concurrentes del tabaco, la exposición a la publicidad y otras prácticas de la industria tabacalera y tendencias seculares.

Además de emitir recomendaciones sobre el tamaño de las etiquetas, el CMCT sugiere que las advertencias "podrán consistir en imágenes o pictogramas, o incluirlos". Esta recomendación debería generalizarse, dada la creciente evidencia de que las advertencias gráficas son más eficaces para llamar la atención de los fumadores y promover el abandono del hábito en comparación con aquellas etiquetas que sólo contienen texto. ${ }^{8,13,14}$ Por ejemplo, Canadá fue el único país participante de la ITC que incluyó las etiquetas gráficas de advertencia antes de la medición basal. Entre los fumadores de países de altos ingresos, los canadienses son los que muestran una mayor tendencia a leer o prestar atención a las etiquetas y han informado que éstas los han inclinado a no fumar un cigarrillo.* No

\footnotetext{
* Hammond D, Fong GT, McNeil A, Borland R, Cummings KM. The effectiveness of cigarette warning labels in informing smokers about the risks of smoking. Tobacco Control. Documento no publicado.
} 


\section{Cuadro III \\ Resumen de los hallazgos del pRoyecto ITC}

Política

Etiquetas de advertencia
- Aumentar el tamaño de la etiqueta hace que la advertencia sea más sobresaliente y notoria para los fumadores*

- El contenido de la etiqueta de advertencia incrementa el conocimiento específico sobre las consecuencias para la salud del hábito de fumar*

- La etiqueta de advertencia propicia que los fumadores piensen en dejar de fumar *

- La etiqueta de advertencia gráfica parece tener un mayor efecto que las etiquetas que contienen sólo texto*

- Las etiquetas de advertencia parecen tener mayor efecto en países de ingreso medio y menor en los de ingreso alto

Prohibición del uso de las

- La prohibición de descripciones ocasiona una disminución de la percepción de los fumadores en relación con los cigarrillos ligeros o suaves, ya que los consideran menos dañinos que los cigarrillos comunes ${ }^{4}$

Prohibiciones relacionadas con - El cumplimiento de una legislación integral libre de tabaco puede lograrse cuando ésta se acompaña de campañas de el hábito de fumar previsión de argumentos que contrarresten la oposición a la reglamentación ${ }^{\ddagger}$

- Las prohibiciones integrales de fumar no propician que los fumadores cambien sus hábitos sino que no decidan fumar en sus hogares, en donde expondrían a los miembros de la familia al humo del tabaco $\$$

- Las proscripciones de fumar en lugares públicos promueven el establecimiento voluntario de prohibiciones en los hogares $\$$

- Las prohibiciones de fumar reciben apoyo por parte de los mismos fumadores en el caso de los lugares públicos de trabajo, incluidos restaurantes y bares ${ }^{\ddagger}$

- Las prohibiciones de fumar promueven el abandono del hábito y ayudan a los fumadores a no reincidir\$§

Impuestos y precio - La evasión de impuestos varía considerablemente de un país a otro\#

- La evasión de impuestos es más común en hombres jóvenes, no blancos y con mayores ingresos que fuman más cigarrillos al día

- La adquisición de cigarrillos en lugares más económicos o libres de impuestos tiene una débil relación con una baja probabilidad de dejar de fumar\#

Prohibiciones en la publicidad - Las prohibiciones integrales en la publicidad pueden disminuir la exposición de los fumadores a las influencias que promueve el tabaco

Regulación del producto

- Los contenidos de alquitrán, nicotina y monóxido de carbono son similares entre la mayoría de las marcas líderes, a pesar del uso de descripciones como "suave", "ligero," o "ultraligero"5

- Los países que utilizan mezclas de tabaco Virginia tienen niveles de nitrosamina específica del tabaco significativamente menores que los países que emplean la mezcla de tabaco americana, que contiene tabaco Burley ${ }^{5}$

- Establecer un nivel mínimo de toxinas en un cigarrillo mediante pruebas ISO estándar no resulta eficaz, ya que las empresas tabacaleras incrementan entonces la ventilación del filtro, un cambio en el diseño que los fumadores compensan aumentando el volumen de sus bocanadas ${ }^{15, *}$

* Hammond D, Fong GT, McNeil A, et al. The effectiveness of cigarette warning labels in informing smokers about the risks of smoking. Tob Control. Documento no publicado

‡ Fong GT, Hyland A, Borland R, et al P. Reductions in tobacco smoke pollution and increases in support for smoke-free public places following the implementation of comprehensive smoke-free workplace legislation in the Republic of Ireland: findings from the ITC Ireland/UK survey. Tob Control. Documento no publicado

$\S$ Borland R, Yong H-H, Cummings KM, et al. Determinants and consequences of smoke-free homes: findings from the ITC collaboration. Tob Control. Documento no publicado

\# Hyland A, Laux F, Higbee C, et al. Cigarette purchase patterns in four countries and its relationship with cessation: findings from the International Tobacco Control Policy Evaluation Survey. Tob Control. Documento no publicado

\& Harris F, MacKintosh AM, Anderson S, et al. The effects of the advertising/promotion ban in the United Kingdom on awareness of tobacco marketing: findings from the International Tobacco Control Policy Four Country Survey. Tob Control. Documento no publicado

* Hammond D,Wiebel F, Kozlowski LT, et al. Revising the ISO smoking machine regime for cigarette emissions: implications for tobacco control policy. Tob Control. Documento no publicado

obstante, las personas que han respondido al ITC en Tailandia y Malasia tenían una mayor tendencia a ver las indicaciones en los paquetes, a pesar de que ningún país había introducido advertencias con fotografías en ellos al momento de efectuar la encuesta de línea de base. ${ }^{3}$ Sin embargo, las advertencias en los paque- tes pueden ser una herramienta educativa más poderosa en naciones de ingresos medianos y bajos en los que existen pocas campañas educativas. La utilización de imágenes para expresar las consecuencias del hábito de fumar puede ser una medida educativa en particular eficaz en lugares donde una parte considerable 
de la población es analfabeta y, por lo tanto, es poco probable que responda o entienda advertencias basadas sólo en texto.

La información obtenida en Brasil sugiere que es probable extrapolar estos resultados del estudio a otros países de Latinoamérica, como parte del programa integral para el control del tabaco. Brasil impuso la utilización de etiquetas de advertencia con gráficos que cubren la superficie total posterior de la cajetilla de cigarrillos en 2002 y las imágenes se cambiaron en 2003. Puesto que estas advertencias se introdujeron junto con otras medidas relacionadas con el tabaco, es difícil determinar en qué medida contribuyeron de manera específica en la subsiguiente disminución del consumo del tabaco. Pese a ello, el número de llamadas a las líneas de apoyo para dejar el cigarrillo se incrementó en grado considerable cuando se introdujeron nuevas etiquetas y $93 \%$ de las personas que llamaron a la línea de apoyo informó que conocía el número telefónico porque lo leyó en ellas. Además, de acuerdo con una encuesta realizada en fumadores poco después de la circulación de las etiquetas, dos terceras partes de ellos señalaron que las imágenes aumentaban el deseo de interrumpir el hábito. ${ }^{14}$ Junto con estudios efectuados en otros países, su éxito aparente en Brasil ha llevado a su aplicación en Venezuela (2004) y Uruguay (2006). Sin embargo, las conclusiones observadas en Brasil se fortalecerán cuando los estudios consideren los cambios individuales en variables específicas de las políticas antes y después de la inclusión de las etiquetas de advertencia. Asimismo, los efectos de las características de éstas provienen sobre todo de comparaciones transversales o la investigación cualitativa y, por lo tanto, se requieren estudios longitudinales. A este respecto, el proyecto ITC tiene la capacidad de evaluar directamente el efecto relativo del contenido gráfico gracias a un análisis longitudinal de datos llevado a cabo antes de la aplicación de las políticas de advertencia gráfica en Australia (2006), un país de ingresos altos, y Tailandia (2005), una nación de ingresos medianos.

Se necesitan este tipo de análisis para aportar el sustento que lleve a recomendar las etiquetas de advertencia. Por ejemplo, pueden analizarse las modificaciones suscitadas a largo plazo y su influencia en el interés de los fumadores después de que éstos se habitúan a las advertencias con el paso del tiempo. Es menos probable que los consumidores se acostumbren a dichas etiquetas con elementos gráficos o una serie rotativa de mensajes en los paquetes de cigarrillos. ${ }^{16} \mathrm{Los}$ análisis longitudinales de datos ITC servirán para reconocer si las distintas reglamentaciones de las etiquetas de advertencia tienen "curvas de desgaste" más amplias o estrechas. Los estudios de estos y otros as- pectos que utilizan información de contextos diversos, nacionales y regionales, contribuirán de modo más amplio a conformar los datos que determinen sus características especiales; de esa manera es posible aprovechar mejor esta medida de bajo costo.

\section{Ambientes libres de humo de tabaco}

El artículo 8 del CMCT promueve medidas políticas para proporcionar "protección contra la exposición al humo de tabaco en los lugares de trabajo interiores, medios de transporte público, lugares públicos cerrados, y, según proceda, otros lugares públicos". Tales lineamientos se crearon con la intención de proteger a los no fumadores de los riesgos de la exposición al humo del tabaco, que en Estados Unidos de América causa 3000 muertes por cáncer de pulmón y entre 35000 y 62000 muertes por enfermedades coronarias del corazón en adultos no fumadores. ${ }^{17,18}$ En ese país, la exposición al humo de tabaco ambiental también se relaciona con la aparición de 8 000 a 26000 nuevos casos de asma en niños y 150000 a 300000 de bronquitis y pulmonía en niños de 18 meses o menos (7500 a 15000 requieren hospitalización). ${ }^{19}$ Las medidas para el ambiente libre de tabaco no sólo protegen a los no fumadores de la exposición involuntaria al cigarrillo sino que favorecen también una conducta de abandono del hábito entre los fumadores..$^{20}$

La industria tabacalera ha combatido de forma activa y ha atenuado con éxito las leyes antitabaco en Latinoamérica, ${ }^{21,22}$ por ejemplo, la mayoría de los países de esa región tiene legislaciones débiles que sólo prohíben fumar en unos cuantos lugares públicos. ${ }^{23} \mathrm{En}$ el caso de México no se puede fumar en edificios del gobierno federal, los que albergan oficinas gubernamentales, lugares donde se otorgan servicios oficiales y en todos los hospitales y clínicas (públicas y privadas) que forman parte del Sistema Nacional de Salud. Se permite fumar en estos edificios siempre que el área de fumar esté delimitada, aislada de las áreas de trabajo y cuente con ventilación o un sistema de purificación del aire. ${ }^{24}$ De igual modo, está prohibido fumar en vuelos que duren menos de 90 minutos. La legislación mexicana existente es relativamente débil, al igual que en la mayor parte de los países latinoamericanos. En Uruguay entró en vigor una legislación integral para los espacios de trabajo libre de tabaco en marzo de 2006 y se espera que otras naciones sigan ese ejemplo.

Pese a la necesidad de una legislación más estricta, cumplir con la legislación existente parece un obstáculo importante para muchos países latinoamericanos. La vigilancia de la concentración de nicotina en las partículas de aire de lugares públicos en muchas ciudades de América Latina indica la presencia de un amplio pa- 
trón de exposición al humo del tabaco en lugares públicos, no obstante la existencia de una legislación que restringe o prohíbe el hábito de fumar. ${ }^{25}$ Por ejemplo, en un estudio reciente realizado en espacios públicos de la Ciudad de México se detectaron partículas de nicotina en casi $80 \%$ de los hospitales, 93\% de las oficinas gubernamentales y $100 \%$ de los espacios en el aeropuerto, restaurantes y bares. ${ }^{26}$ En realidad, este incumplimiento de las leyes acentúa el problema de la debilidad general de la reglamentación para áreas de trabajo libres de humo de tabaco en América Latina. ${ }^{23}$

El cumplimiento de las prohibiciones integrales se promueve por medio de campañas educativas que crean conciencia del peligro que supone el humo de segunda mano y proporcionan a las personas argumentos para favorecer la legislación y anticipar la oposición a ésta. En Irlanda, por ejemplo, dichas campañas de sensibilización ayudaron, al parecer, a asegurar el respeto a la prohibición integral de no fumar.* El análisis de los datos del proyecto ITC de Irlanda reconoce un descenso notable entre los fumadores que fumaron la última vez que estuvieron en un restaurante (de 85\% antes de la prohibición a 3\% después de ella), un bar (de 98 a 5\%) o el lugar de trabajo (de 62 a 14\%). ${ }^{*}$ Como se ha documentado en otros países, ${ }^{27,28}$ el apoyo de los fumadores irlandeses a las leyes para contar con ambientes libres de humo de tabaco se incrementó después de la prohibición, más incluso en comparación con la cohorte de fumadores del Reino Unido. Por lo tanto, parecería que el costo político es muy bajo o que no existe en términos del apoyo público. Además, estas conclusiones sobre el cumplimiento de estas directrices en Irlanda ilustran una ventaja clave para el ITC: las interferencias causales relacionadas con el efecto de la reglamentación se fortalecen si se dispone de datos que documenten los cambios, más allá de cualquier tendencia identificada en cohortes comparables de otros países, en los cuales dicha legislación no está en vigor.

Los datos del ITC en Irlanda también se suman al corpus de conocimientos acerca de la influencia de las políticas en los ambientes libres de humo y la conducta de abandono.* Casi todas las personas entrevistadas que habían dejado de fumar durante el seguimiento mencionaron que la prohibición del cigarrillo les había ayudado a abandonar el hábito (80\%) y permanecer así

\footnotetext{
* Fong GT, Hyland A, Borland R, Hammond D, Hastings G, et al. Reductions in tobacco smoke pollution and increases in support for smoke-free public places following the implementation of comprehensive smoke-free workplace legislation in the Republic of Ireland: Findings from the ITC Ireland/UK survey. Tobacco Control. Documento no publicado.
}

(88\%). De manera adicional, casi la mitad (46\%) de las personas que lo continuaron informó durante el seguimiento que la prohibición hacía más probable que dejaran de fumar. De estos fumadores, una gran proporción evitó los bares (35\%) y los restaurantes (18\%) por la prohibición. Los opositores a las leyes de ambientes libres de humo han aducido que la limitación de fumar en lugares públicos podría desplazar el hábito a los hogares particulares, en donde los miembros de las familias podrían experimentar una mayor exposición al humo de tabaco en el ambiente. No obstante, después de la prohibición se advirtió un descenso notorio del porcentaje de fumadores irlandeses que permitían fumar en casa ( 85 a 80\%).

Estos resultados son consistentes con otros estudios, ${ }^{29,30}$ incluido el análisis de la encuesta ITC en cuatro países, ${ }^{*}$ y revelan que estas políticas llevaron de forma voluntaria a los fumadores a instituir medidas contra el hábito en el hogar. Además, los ambientes libres de humo parecen incrementar el abandono del consumo de cigarrillos y prevenir la reincidencia. ${ }^{* 31}$ Todo esto sugiere que los beneficios de las prohibiciones en los espacios públicos se propagan a su vez a los espacios privados.

A pesar de que las evidencias sobre las consecuencias de las políticas en los ambientes libres de humo son consistentes, por lo general su efecto ha sido limitado por la falta de grupos de control, un seguimiento limitado y medidas de cumplimiento inadecuadas o inexistentes. ${ }^{32}$ Conforme el CMCT promueva en los países latinoamericanos la aplicación de este tipo de directrices integrales, será necesario determinar su observancia para valorar de manera adecuada sus efectos. Investigaciones como el proyecto ITC pueden proporcionar retroalimentación sobre la adopción o cumplimiento de las políticas para ambientes libres de humo en lugares de trabajo, incluidos los restaurantes, cafés y bares, así como el transporte público y los hogares de los fumadores; todas estas disposiciones podrían también informar sobre las maneras de maximizar el cumplimiento de las prohibiciones. A este respecto, los datos de fumadores adultos pueden complementar otros esfuerzos de supervisión, como, por ejemplo, la vigilancia de la exposición al humo de tabaco en la población joven a través de la Encuesta Global para Jóvenes sobre Tabaco, ${ }^{33,34}$ la medición del humo de tabaco en sitios públicos seleccionados ${ }^{25}$ y la intervención de agencias encargadas de seguir y vigilar la aplicación. Son

\footnotetext{
* Borland R, Yong H-H, Cummings KM, Hyland A, Anderson S, Fong GT. Determinants and consequences of smoke-free homes: Findings from the ITC collaboration. Tobacco Control. Documento no publicado.
} 
necesarias las comparaciones entre países de Latinoamérica y otras naciones para enfocarse específicamente en el efecto de las políticas en ambientes libres de humo y los mecanismos a través de los cuales funcionan en los países de ingresos medios y bajos. Estos datos pueden depurar dichas reglamentaciones en naciones en las que se llevan a cabo estudios de evaluación, al tiempo que proporcionan información valiosa a otros países que consideran la utilización de estas medidas.

\section{Precios e impuestos}

El artículo 6 del CMCT solicita a las naciones incrementar el costo de los cigarrillos: "aplicar a los productos de tabaco políticas tributarias y, si corresponde, políticas de precios para contribuir [...] a reducir el consumo de tabaco." Esta política se basa sobre todo en la evidencia de países de ingresos altos en los que un incremento de $10 \%$ del costo real de los cigarrillos precipita una disminución de 3 a 5\% del consumo per cápita del tabaco; los precios más elevados promueven el abandono del hábito y la disminución del uso en consumidores continuos. ${ }^{2,35}$ La información que procede de países de ingresos medios y bajos sugiere que son posibles disminuciones mayores del consumo relacionadas con el incremento de los precios; por ejemplo, un estudio sobre las variaciones del precio en México de 1994 a 2002 reveló que un aumento de 10\% en el impuesto causó un descenso del consumo de $6.2 \%{ }^{36}$ Sin embargo, muchas de las vías a través de las cuales el impuesto o el precio tienen un efecto en la conducta relacionada con el consumo del tabaco todavía son relativamente desconocidas. El proyecto ITC es el primer estudio longitudinal internacional que analiza los efectos de las políticas de precios e impuestos en muestras representativas de fumadores. En consecuencia, permitirá la conducción de un análisis más completo y detallado sobre el efecto del precio en la conducta individual, imposible hasta ahora en estudios anteriores, ya que se ha establecido un seguimiento sobre todo a los cambios del consumo del tabaco per cápita y la prevalencia del hábito por nivel de población.

El precio, la accesibilidad y los impuestos a los cigarrillos varían en grado amplio en Latinoamérica. Por ejemplo, un estudio de precios examinaba los minutos de trabajo necesarios para comprar un paquete de cigarrillos en el año 2000; la investigación concluyó que los cigarrillos Marlboro eran más económicos en Argentina, Brasil, Colombia y Venezuela (20.5, 21.8, 24.5 y 28.5 minutos / paquete, respectivamente) que en Chile, México y Panamá (38.4, 49.4 y 81.4 minutos/paquete, respectivamente).$^{37}$ Las tendencias en el índice del precio de los cigarrillos señalan que, de estos países, sólo Brasil y Panamá lo incrementaron más de 10\% entre 1991 y 2000 (11.05 y 14.33\%, respectivamente).

El proyecto ITC tiene una posición única para analizar una serie de aspectos relacionados con el efecto del impuesto y precio sobre el uso del tabaco, conductas que algunos estudios previos no eran capaces de analizar; las encuestas que implementa recogen información detallada sobre todos los aspectos del proceso de adquisición de cigarrillos (por ejemplo, la compra en diversos puntos de venta, incluidos teléfono e internet), el tipo de tienda donde se adquieren, la ubicación de los expendios (como las tiendas libres de impuestos o las jurisdicciones con impuestos más bajos), el conocimiento y el uso de promociones (por ejemplo, descuentos por compra de paquetes múltiples o con cupones), la cantidad adquirida, y otros más. Esta información detallada puede emplearse para observar las conductas de compensación usadas por los consumidores para contrarrestar el efecto del aumento del impuesto o el precio, o ambas cosas. Por ejemplo, como reacción al incremento de precios los fumadores pueden cambiar a marcas más baratas, comprar en puntos de venta más económicos, adquirir los cigarrillos por paquete o aprovechar promociones que ofrecen rebajas, entre otras estrategias.

La posibilidad de evadir impuestos y el comercio ilícito del tabaco son una cuestión que mencionan con frecuencia los opositores a esta medida. ${ }^{38}$ Los análisis iniciales de ITC relacionados con los precios han examinado el grado en el cual los compradores hacen esfuerzos especiales para evitar los impuestos sobre los cigarrillos, al comprarlos en lugares exentos de impuestos o de baja exacción, incluidas las reservas indígenas (en EEUU), las tiendas libres de impuestos, las comisarías militares, las compras por teléfono o internet, los vendedores independientes (como los puestos callejeros) $u$ otras jurisdicciones que cobran bajos impuestos. * Los datos de la primera medición de Australia, Canadá, el Reino Unido y Estados Unidos de América mostraron una considerable variación en la prevalencia de la evasión de impuestos, desde un bajo porcentaje de 1.0\% en Australia hasta uno alto de $15.7 \%$ en el Reino Unido. Una comparación con los datos de la segunda medición indica que la evasión de impuestos ha cambiado poco con el transcurso del tiempo en Australia, Canadá y Estados Unidos de América, en tanto que se ha incrementado en grado notable en el Reino Unido (20.4\%).

\footnotetext{
* Hyland A, Laux F, Higbee C, Hastings G, Ross H, et al. Cigarette purchase patterns in four countries and its relationship with cessation: Findings from the International Tobacco Control Policy Evaluation Survey. Tobacco Control. Documento no publicado.
} 
Los sitios para adquirir cigarrillos de bajo costo o sin impuestos también variaron en cada país; las reservas indígenas fueron el recurso más común en Estados Unidos, las tiendas libres de impuestos en Australia (y la segunda en el Reino Unido) y los puntos de venta en la frontera en el Reino Unido. A pesar de que a menudo se comenta que internet es un recurso para adquirir cigarrillos a bajo precio, ${ }^{39}$ la información de la primera medición de la ITC indica que menos de $1 \%$ de los fumadores en los cuatro países mencionados ha adquirido cigarrillos por medio de la red y los porcentajes no muestran un incremento de consideración respecto al tiempo.

La posibilidad de incurrir en la evasión de impuestos $u$ otros esfuerzos para compensar el alza del precio de los cigarrillos pueden limitar el efecto de las políticas de precios para atenuar el hábito de fumar. Datos de las dos primeras mediciones del ITC en cuatro países sugieren algunos motivos de preocupación. De manera específica, los fumadores que adquirieron cigarrillos en lugares donde no se cobran impuestos (por lo general los hombres más jóvenes, individuos distintos de la raza blanca y los que fuman más cigarrillos por día) tienen una probabilidad más baja de abandonar el hábito que los fumadores que no consiguen los cigarrillos en esos sitios $(R R=0.80, p=0.09)$. Esta relación fue más sólida en Canadá y Estados Unidos de América.

Con el transcurso del tiempo, la extensa información longitudinal recolectada en el estudio ITC permitirá una comprensión más completa de la respuesta de los fumadores a los cambios en los precios de los cigarrillos, sobre todo en relación con las conductas compensatorias, como cambiar el modo y el lugar de comprar los cigarrillos y la forma de fumarlos (por ejemplo, inhalaciones más profundas y continuas). Mientras se aclara el mecanismo mediante el cual estas respuestas limitan la eficacia de la medida, los análisis de los datos del ITC también proporcionan nueva evidencia sobre las posibles sinergias entre el precio elevado de los cigarrillos y otras políticas antitabaco. Por ejemplo, las disposiciones que tienen una influencia en la aceptación del hábito de fumar, como las leyes que promueven los ambientes libres de humo, pueden interactuar con el incremento de los impuestos, como parece suceder entre los jóvenes con el precio y las normas entre pares ${ }^{40}$ Además, el análisis de datos del ITC, al combinar los países de ingresos bajos, medianos y altos, aportará información vinculada con la existencia de efectos diferenciales sobre el impuesto y el precio, según sean la etapa de desarrollo, la institución de políticas alternativas para frenar el comercio ilícito del tabaco y otros esfuerzos para impedir la evasión de impuestos.

\section{Discusión}

El proyecto ITC constituye un espacio único para ayudar a las naciones a aprender unas de otras de manera colectiva, con la finalidad de frenar la demanda de los productos del tabaco. En consecuencia, es posible abatir las repercusiones económicas y sociales de las enfermedades secundarias al tabaco, en particular aquellas que aparecerán en el mundo en desarrollo en las próximas dos décadas. La presente revisión del marco conceptual, el diseño y los resultados preliminares del proyecto ITC ilustra una posible forma de utilizar estudios similares en países de América Latina, con el fin de acelerar la adopción de intervenciones de control del tabaco basadas en evidencias durante la era del CMCT y en adelante.

Por supuesto, no debe asumirse que las políticas y los programas que funcionan en un país lo harán de la misma manera en otros lugares del mundo. La validez interna requiere siempre confirmación. Por lo tanto, la reciente expansión del proyecto ITC a Asia tiene el propósito explícito de valorar las intervenciones políticas que han sido eficaces en las naciones occidentales. No es posible extrapolar los resultados a las naciones que poseen distintos contextos culturales, sociales y políticos. De la misma manera, el desarrollo adecuado y la consolidación de evidencias para las políticas de control de tabaco más eficaces en México y otras naciones latinoamericanas exigen esfuerzos de evaluación comparables en la región de América Latina. Hasta la fecha, los resultados sugieren que algunas disposiciones pueden ser más eficaces que otras en países de ingresos medianos (como el precio y las etiquetas de advertencia en las cajetillas), pero otras pueden ser menos efectivas, tal vez, en parte, por los retos que acompañan al cumplimiento de las reglamentaciones. Por consiguiente, los análisis comparativos resultantes podrían determinar si los contextos sociales de Latinoamérica tienen particularidades que ejercen una influencia distinta sobre los lineamientos y los programas de control de tabaco que funcionan, así como sus razones para funcionar.

Un estudio de evaluación del proyecto ITC podría ayudar a los generadores de políticas y los profesionales de la salud pública en estos países a vigilar y comprender el efecto de las intervenciones en su particular contexto nacional. El proyecto proporciona un marco teórico y metodológico para el tipo de estudios de evaluación que contribuirían a depurar y promover la adopción de directrices eficaces contra el consumo del tabaco. La evaluación coordinada en el plano internacional de políticas recomendadas por el CMCT (por primera vez el empleo de un mecanismo único, un tra- 
tado, para resolver un asunto de salud global) proporcionará también valiosas lecciones sobre la utilidad y la forma de mejorar este tipo de acuerdos para combatir otras amenazas a la salud pública global.

\section{Agradecimientos}

La investigación fue auspiciada con fondos del Instituto Nacional de Cáncer de EUA/NIH (del Centro Transdisciplinario de Investigación en el Uso del Tabaco [TTURC], P50 CA111236, R01 CA100362, y el Programa de Educación y Desarrollo de Carreras Relacionadas con el Cáncer, R25 CA 057699), los Institutos Canadienses de Investigación en Salud, el Consejo Nacional de Salud e Investigación Médica, el Departamento Público Australiano de Salud y Envejecimiento, Investigación sobre Cáncer en el Reino Unido, el Centro de Investigación en Conductas y Evaluación de Programas del Instituto Nacional del Cáncer en Canadá, La Sociedad Canadiense para el Cáncer y la Iniciativa Canadiense para la Investigación sobre el Control del Tabaco.

\section{Referencias}

I.World Health Organization. Framework Convention on Tobacco Control. Ginebra, Suiza:WHO, Tobacco Free Initiative, 2003. 2. Chaloupka FJ, Hu TW,Warner KE et al.The taxation of tobacco products. In: Chaloupka FJ, Jha P, ed. Tobacco control in developing countries. Oxford: Oxford University Press, 2000;237-272.

3. Maizurah O, Kin F, Borland R et al. Initial findings from the South East Asia Tobacco Control Policy Survey. East-West Conference on Tobacco and Alcohol. Pasadena, California, 2005.

4. Fong GT. Evaluating the effects of the september 2003 European Union policy banning "light/mild" cigarette brand descriptors: findings from the International Tobacco Control Policy Evaluation Survey. Health Care Can 2005.

5. Polzin GM, Vaughan CL, Tavakoli AD et al. Analysis of physical characteristics and smoke chemistry of before and after changes in regulation.TTURC Symposium:Transdisciplinary Tobacco Use Research from Laboratory to the Population. Bethesda, Maryland: National Institutes of Health, 2005.

6. Institute of Medicine. Speaking of health: assessing health communication strategies for diverse populations. Washington DC: National Academy of Sciences, Institute of Medicine, 2002.

7. Kottler P, Roberto N, Lee N. Social marketing: improving the quality of life. 2a ed. Thousand Oaks, California: SAGE, 2002.

8. Strahan E, White KS, Fong GT et al. Enhancing the effectiveness of message labels on tobacco packaging: a social psychological perspective. Tob Control 2002;1 I:183-190.

9. Manhood G. Warnings that tell the truth: breaking new ground in Canada. Tob Control 1999;8:356-361.

10. Borland R. Tobacco health warnings and smoking-related cognitions and behaviors. Addiction 1997;92(II): 1427-1435.

II. Kaiserman MJ.The effectiveness of health warning messages. Tob Control 1993;2:267-269.

12. Fong GT, Hammond D, Borland R et al. Quasi-experimental evaluation of the enhancement of warning labels in the United Kingdom: initial findings from the International Tobacco Control Policy Evaluation Survey. Conferencia en Society for Research on Nicotine and Tobacco. Scottsdale, Arizona, USA, 2004.

13. Hammond D, Fong GT, MacDonald P et al. Impact of graphic canadian warning labels on adult smoking behavior.Tob Control 2003;12:39|-395. 14. Cavalcante TM. Labelling and packaging in Brazil. Ginebra, Suiza: World Health Organization, 2003.

15. O'Connor RJ, McNeil A, Comming KM, Kozlowski LT, Giovino GA. How did UK cigarette makers get their brands to $10 \mathrm{mg}$ "tar" or less? BMJ 2006;332:302.

16. USDHHS. Reducing the health consequences of smoking: 25 years of progress. A report of the surgeon general. Bethesda, Maryland: US Department of Health and Human Services, 1989.

17. Center for Disease Control and Prevention. Annual smokingattributable mortality, years of potential life lost, and economic costsUnited States, 1995-1999. MMWR Morb Mortal Wkly Rep 2002;5I(I4):300-303.

18. $\mathrm{NCl}$. Health effects of exposure to environment tobacco smoke. Smoking and tobacco control monograph No. I0. Bethesda, MD: U.S Department of Health and Human Services, National Institutes of Health, National Cancer Institute, 1999.

19. EPA. Respiratory health effects of passive smoking: lung cancer and other disorders. Washington, DC: United States Environmental

Protection Agency, 1992

20. Fichtenberg CM, Glantz SA. Effect of smoke-free policies on smoking behavior: a systematic review. Br Med J 2002;325(7357): 188.

21. Sebrié EM, Barnoya J, Pérez-Stable EJ et al. Tobacco industry successfully prevented tobacco control legislation in Argentina. Tob Control 2005; 14(5):e2.

22. Barnoya J, Glantz SA. Tobacco industry success in preventing regulation of secondhand smoke in Latin America:The "Latin Project". Tob Control 2002; I I (4):305-3I4.

23. Organización Panamericana de la Salud. 132a Sesión del Comité Ejecutivo.Washington, DC: , OPS,OMS, 2003.

24. Ibáñez-Hernández NA. Disposiciones jurídicas federales sobre la prohibición de fumar. En:Valdés Salgado R, Lazcano-Ponce EC, HernándezAvila $\mathrm{M}$, ed. Primer informe sobre el combate al tabaquismo. Cuernavaca, Morelos, México: Instituto Nacional de Salud Pública, 2005; I4I-I47. 25. Navas-Acien A, Peruga A, Breysse $P$ et al. Secondhand tobacco smoke in public places in Latin America, 2002-2003. JAMA 2004;29I(22):274I-2745. 26. Navas-Acien A, Valdés-Salgado R. Niveles de nicotina en el ambiente de lugares públicos y de trabajo del Distrito Federal. En:Valdés Salgado R, Lazcano-Ponce EC, Hernández-Avila M, ed. Primer informe sobre el combate al tabaquismo. Cuernavaca, Morelos: Instituto Nacional de Salud Pública, 2005.

27. Tang H, Cowling D, Lloyd J et al. Changes of attitudes and patronage behaviors in response to a smoke-free law. Am J Public Health 2003;93:6 II-6I7.

28. Gilpin EA, Lee L, Pierce JP. Changes in population attitudes about where smoking should and should not be allowed: California versus the rest of the USA. Tob Control 2004;13:38-44.

29. Borland R, Mullins R, Trotter $L$ et al. Trends in environmental tobacco smoke restrictions in the home. Tob Control 1999;8:266-27I.

30. Merom D, Rissel C. Factors associated with smoke-free homes in NSW: results from the 1998 NSW Health Survey. Aust N Z J Public Health 200I;25(4):339-345.

31. Pizacani B, Martin J, Stark M et al.A prospective study of household smoking bans and subsequent cessation related behavior: the role of stage of change. Tob Control 2004;13:23-28.

32. Borland R, Hau HY, Fong GT et al. Restrictions on smoking across four countries: initial findings from the International Tobacco Control Policy Evaluation Survey. Madison, Wisconsin: Society for Research on Nicotine and Tobacco, 2004 
33. Martin JP, Peruga A. The global youth tobacco survey: results in the Americas. Epidemiol Bull 2002;23(2):6-9.

34. CDC. Encuesta Mundial sobre Tabaquismo en Jóvenes. Atlanta: Centers for Disease Control and Prevention, World Health Organization, 2005.

35. Chaloupka FJ,Warner KE. The economics of smoking. En: Cuyler AJ, Newhouse JP, ed. Handbook of economics. Amsterdam: Elsiever, 2000.

36. Sesma-Vásquez S, Pérez-Rico R, Puentes-Rosas E et al. El precio como determinante del consumo de tabaco en México, 1994-2002. En: Valdés Salgado R, Lazcano-Ponce EC, Hernández-Avila M, ed.

Cuernavaca, Morelos: Instituto Nacional de Salud Pública, 2005;125-1 32.

37. Guinden GE, Tobin S, Yach D. Trends and affordability of cigarette prices: ample room for tax increases and related health gains. Tob Control 2002; II:35-43.
38. Jha P, Chaloupka FJ. Curbing the epidemic: governments and the economics of tobacco control. Washington, DC: The International Bank for Reconstruction and Development, 1999.

39. Knowles JHJ, Wanke KL, Kawachi I. Internet sales of tobacco: heading off the new e-pidemic.J Public Health Policy 2004;25(2): 162-72. 40. Powell LM, Tauras JA, Ross H.The importance of peer effects, cigarette prices and tobacco control policies for youth smoking behavior.J Health Econ 2005;24:950-968. 